\title{
Dirk van Haren-Noman
}

Am $\delta$. März d. J. ist im Alter von 41 Jahren zu Amsterdam ein aus-gezeichneter Fachgenosse aus dem Leben geschieden. Geehrt von seinen Collegen, voifi Patienten und Schülern geliebt, schien ihm noch ein reiches Wirken und Werden bevorzustehen. Er hat wenig länger als ein Jahrzehnt der Dermatologi $\beta$ angehört, und doch kannte Jeder seinen geachteten Namen. In Batavia geboren,

Mitteilungen

vollendete er in Holland seine Erziehung, wurde schon als Schüler seiner hervvr-ragenden Begabung halber bemerkt und wandte sích zunächst dem Studium der beschreibenden Naturwissenschaften zu, damit unbewusst und zuíällig der besten Vorbereitung far seinen späteren Beruf hingegeben. Er beschäftigte sicli nament-lich an der niederländischen zovlogischen Station zu Vlissingen, machte eine wisscnschaftliebe Nordsee-Exploration mit und wurde beauftragt, die auf der Folarreise des „Willem Barends” gesammelten Mollusken zu bearbeiten. Als diese Arbeit im Niederländischen Archiv für Zoologie erschien, war er bereits zur Medicin übergegangen. Hier promvvierte er im Jahre 1881 mit einer experi-mentellen Untersuehung über die anatomiscben Veriinderungen nach Immübilisation der Gelenke und wurde Assistent des pathologisehen Anatomen Mac Gillavry. Als solcher publicieite er Arbeiten tiler gelbe Leberatropbie, fiber die Todes-ursache nach Hautverbrennungen und über Lepra. Mittlerweile zum Lector der Anatomie an der Universität Utrecht ernannt, beschäftigte er sich mit der Histo-logie der Haut und wurde dadurch dem Jnteresse für Dermatologie näher geführt. Meisterhaft geschult in alien Zweigen microscopischer Technik, erzielte er grossen Erfolg mit seinen Vorlesungen über dermatologische Gewebelehre, bildete sich dann an ausländischen Klíniken weiter aus und wurde im Jahre 1886 zum Professor für Hautkrankheiten und Syphilis und Director der Klinik zu Amsterdam gewählt. Hier arbeitete er unausgesetzt weiter und legte das Hauptgewicht auf die Lehrthätigkeit und klinische Beobachtung. Sein weitbekannter Atlas giebt von der Scharfsichtigkeit seiner Auffassung und Perceptionsenergie Zeugnis. Jedes Wort ist durchdacht, jedes Bild treu, jede Wendung schlicht und wahr. Vielfach iiegt wenigen Zeilen ein umfassendes Studium der Litteratur zu Grunde, überall tritt das Bestreben zu Tage, nicht aus Wenigem Viel, sondern aus Vielem etwas Gediegenes zu gestalten. So war er auch als Mensch. Ein ernster, gütiger Mann, ein geschlossener Charakter. eine Persönlichkeit ist mit ihm dahin. L. 